\title{
Against the Empirical Viability of the Deutsch-Wallace-Everett Approach to Quantum Mechanics
}

\author{
Richard Dawid, Karim Thébault \\ Munich Center for Mathematical Philosophy, Ludwig Maximilians Universität, \\ Ludwigstrasse 31, D-80539, Munich, Germany \\ richard.dawid@univie.ac.at, karim.thebault@gmail.com
}

\begin{abstract}
The subjective Everettian approach to quantum mechanics presented by Deutsch and Wallace fails to constitute an empirically viable theory of quantum phenomena. The decision theoretic implementation of the Born rule realized in this approach provides no basis for rejecting Everettian quantum mechanics in the face of empirical data that contradicts the Born rule. The approach of Greaves and Myrvold, which provides a subjective implementation of the Born rule as well but derives it from empirical data rather than decision theoretic arguments, avoids the problem faced by Deutsch and Wallace and is empirically viable. However, there is good reason to cast doubts on its scientific value.
\end{abstract}

Keywords: Everett interpretation, Many worlds, Empirical Viability

\section{Introduction}

The last decade of debate with regard to the interpretation of quantum theory has seen the ascendance of a particular variant of the Everettian approach, purported to be free of the problems with probability that beset its forebears. The essential step in these neo-Everettian approaches comes from an idea first presented by David Deutsch and then explicated and carried further by David Wallace. They suggested that a satisfactory Everettian 
interpretation of quantum mechanics could be based on a purely subjective 1 emergence of the probabilistic role of the wave function. According to this understanding, the connection between the wave function and probabilities does not rely on any objective characteristics of the many worlds scenario (be it objective probabilities, numbers of branches or other) but is rather a product of the rational behaviour of agents who find themselves in one branch of the Everettian universe. Deutsch Deutsch (1999) and later Wallace Wallace $(2007,2009,2012)$ demonstrated that, provided that Everettian quantum mechanics was true, the Born rule could be established under certain conditions as being constitutive of any rational betting behaviour on future outcomes of quantum experiments.

This Deutsch-Wallace-Everett (DWE) approach to quantum theory has not been without its critics. One line of critical response found within the literature focuses upon the the structure of the decision theoretic proof itself, and relies upon either disputing the notion of rationality Price (2010), or challenging the derivation of the Born rule as a unique/viable subjective probabilistic measure Lewis (2006); Hemmo and Pitowsky (2007). Such internal criticisms rest upon implicitly accepting the viability of the DWE framework but questioning particular aspects of its implementation. Another important line of criticism seeks to undermine the DWE claim of a derivation Born rule on the basis of a decoherence related circularity objection Zurek (2005, 2010); Baker (2007); Kent (2010).

In the present paper, we will not address the question of the internal cogency of the selection of rationality criteria nor the problems related to the decoherence argument. Rather, we will argue that, even if these two points of criticism are disregarded, the Deutsch-Wallace-Everett programme is afflicted by a further exogenous malady owing to a failure to fulfil an essential condition for being a satisfactory scientific approach: We will claim that the DWE scheme is fundamentally flawed since it does not offer a basis for rejecting Everettian quantum mechanics in the face of evidence that contradicts the predictions of quantum mechanics.

We begin in Section $\S 2$ by briefly outlining the key conceptual steps taken within the DWE approach towards quantum mechanics. This programme is

\footnotetext{
${ }^{1}$ Here the use of 'epistemic' rather than 'subjective' would perhaps be more consistent with the terminology in use within the philosophy of science. However, we will retain the original terminology to avoid confusion
} 
then, in Section $\S 3$, shown to fail to be empirically viable according to our definition. In $\S 4$ we consider a strategy that has been proposed to deal with the issue of predictions/evidence by Greaves and Myvold Greaves and Myrvold (2010). They believe we should explicitly ascribe (non-probabilistic) primitive weights to branches according to the Born rule and then base this branch weighting rule upon the empirical evidence we have hitherto taken to refer to probabilities. We will point out that this strategy departs from DWE in precisely the respect that any Everettian is at pains to avoid: it introduces a new rule in order to provide an interpretation of the wave function. Furthermore, it will be argued that this additional structure is of questionable scientific value, and thus that the Greaves-Myvold approach constitutes a rather unattractive interpretational option.

\section{The Deutsch Wallace Approach}

Let us briefly consider the line of reasoning presented by Deutsch and Wallace. Their starting point is a core problem of the Everettian approach. Consider an agent who carries out a quantum experiment that can have a number of different outcomes. Everettian QM relates each outcome to one branch of the Everettian universe. Since an actual instantiation of our agent exists in each of those branches, the agent's chances of finding themselves in one specific branch after the experiment might be expected to be directly determined by the number of branches which fork off from the initial state of the experiment. Closer analysis shows, however, that such branch countingin conjunction with the Everettian understanding that the branches emerge based on decoherence without additional assumptions - cannot coherently reproduce the probabilities provided by quantum mechanics. (See e.g. Rae (2009)).

The Deutsch-Wallace strategy Deutsch (1999); Wallace (2007, 2009, 2012) is to deal with this problem by deploying a double strategy. First, they argue that it is impossible in principle to extract probability statements on the outcomes of quantum experiments on the basis of branch counting. Since decoherence is fuzzy in the sense that there is no precise way of telling at what point in time decoherence occurs, it is impossible to specify one definitive branching structure for a quantum process. ${ }^{2}$ This in turn implies that no

\footnotetext{
${ }^{2}$ We will in this paper neglect the issues inherent within any Everettian invocation of a decoherence based ontology.
} 
definitive probabilistic conclusions can be drawn from branch counting. The DWE approach is then based upon the conclusion that quantum probabilities cannot be implemented at an objective level in an Everettian framework at all. The many worlds structure thus must not be understood in terms of a discrete set of branches where the probability for measuring a specific result in a quantum experiment can be extracted from the number of alternative branches which fork off during the experiment. Under the DWE approach it is then claimed that, once the connection between measurement probabilities and the branching structure is broken, the problem of conceptual incompatibility between the two is thereby dissolved.

It remains to be shown how measurement probabilities are related to Everettian QM at all. In a second step, the DWE approach leads us to implement probabilities at a subjective level by introducing a decision theoretic analysis. We ask the question as to which rational betting strategies are open to an agent living in a branch of the Everettian world; and then postulate rationality criteria which are taken to be necessary for coherent reasoning. On that basis, the claim is that one can show that, up to linear transformations, applying the Born rule to the amplitudes provided by quantum mechanics constitutes the only rational betting strategy if one is convinced that Everettian QM is true. ${ }^{3}$ The viability of Everettian quantum mechanics as an adequate description of nature thus is claimed to follow from the rationality restriction on our betting behaviour.

The implementation of the decision theoretic argument within the DWE scheme has the following form:

A1: The Everettian perspective on quantum mechanics amounts to nothing more than literally accepting the equations of quantum mechanics (which, in of themselves, do not imply the probabilistic implementation of quantum mechanics based on the Born rule).

A2: For an agent who understands themselves as part of an Everettian universe, the Born rule constitutes the only possible basis for rational betting on the outcome of quantum processes. If Everettian QM is true, a rational

\footnotetext{
${ }^{3}$ As noted above many questions have been raised with regard to the cogency of the rationality criteria chosen. We will not add to the already substantial literature in this line.
} 
scientist thus should apply the Born rule as a probabilistic measure for possible experimental outcomes.

$\Rightarrow$ C1 Everettian QM predicts that outcomes of quantum experiments adhere to the Born rule.

O: The predictions which can be extracted from Everettian QM by deploying the Born rule are empirically confirmed by all availlable data.

$\Rightarrow$ C2: Everettian QM must be taken to be a viable description of quantum phenomena at this point.

In the following, we take observation $\mathbf{O}$ for granted and, for the sake of the argument, concede assumptions $\mathbf{A} \mathbf{1}$ and $\mathbf{A 2}$. We will argue on that basis that the Deutsch-Wallace argument is fallacious and leads to a misrepresentation of the actual status of Everettian QM. The crux of the DWE argument lies in C1. As we will see, calling the fact that rationality enforces a specific betting behaviour a prediction is highly misleading since it does not accord with the standard meaning of scientific prediction and is too weak for establishing a scientific theory's empirical viability.

\section{The Problem of Empirical Viability}

To understand the problem faced by the Deutsch-Wallace approach, we must be clear about the role Everettian QM is supposed to play. Considering the currently available data, the canonical interpretation of quantum mechanics (henceforth called QM) provides an empirically fully viable basis for calculating non-relativistic quantum phenomena. The conceptual difficulties of QM do not amount to empirical deficits. Therefore, we expect Everettian QM to resolve the conceptual difficulties of QM while retaining the empirically confirmed implications of QM. If Everettian QM fulfils that condition, we can call it empirically viable with respect to the current empirical status quo. We know, however, that Everettian QM is not fully empirically equivalent with QM since it allows for recoherence effects which are absent in QM. Barrett (2011) In order to check the empirical viability of Everettian QM we thus have to demand empirical equivalence with respect to the available data while allowing for empirical differences with respect to data that lies beyond the range of the experiments carried out up to this point. In other words, we 
must require that Everettian QM meets the standard of empirical viability set by QM with respect to the experiments carried out up to this point.

In the following, we will analyse whether or not Everettian QM as understood by Deutsch and Wallace can be called an empirically viable theory in the described sense. To that end, let us further specify the conditions which have to be fulfilled in order to acknowledge that a scheme $\mathbf{S}$ meets the standard of empirical viability set by some theory $\mathbf{T}$. We can immediately state a simple condition that is necessary without any doubt:

$\mathbf{I}_{1}$ : A scheme $\mathbf{S}$ meets the standard of empirical viability set by a theory $\mathbf{T}$ only if $\mathbf{S}$ is not refuted by the collected empirical data that confirms $\mathbf{T}$.

This condition seems fulfilled by the Deutsch-Wallace-Everett (DWE) approach to quantum mechanics. Given that recoherence effects are too small for being observable in present day experiments, Everettian QM does not contain any empirical implications at variance with QM that can be contradicted by the available empirical data. Thus so far as $\mathbf{I}_{1}$ goes DWE has no problems. However, a simple example can show that this condition fails to exclude all classes of theories that are obviously not empirically viable.

Let us consider Newtonian mechanics and compare it to a scheme SN that assumes that objects move freely in space without following any deterministic or probabilistic equations of motion. According to $\mathbf{I}_{1}$, we could say that this theory meets the standard of empirical viability set by Newtonian mechanics. After all, since $\mathbf{S N}$ gives no predictions regarding the movements of objects at all, it cannot offer any predictions which contradict Newtonian mechanics. It is obviously absurd, however, to say that SN meets the standard of empirical viability set by Newtonian physics, and there is a clear reason why: if objects were found to move in space in disagreement with Newton's laws, that would contradict Newtonian physics but would not contradict SN. Therefore, we clearly can distinguish empirically between the two schemes and must call Newtonian mechanics the by far stronger theory.

In order to exclude examples like the one presented above, we thus need one more condition. A very plausible choice is:

$\mathbf{I}_{2}$ : A scheme $\mathbf{S}$ meets the standard of empirical viability set by a theory $\mathbf{T}$ only if any data that could have been collected in experiments which have been carried out in the past and would have refuted theory $\mathbf{T}$ would also have refuted $\mathbf{S}$. 
The question is then whether or not the DWE approach to quantum mechanics satisfies $\mathbf{I}_{2}$.

As has been noted in the literature (see e.g. Kent (2010)), the DWE approach does not give us an objective basis for predicting the results of quantum measurements. Any outcome of a quantum process that does not contradict non-statistical laws is represented by a branch in the many worlds structure - and since all branches in a many worlds scenario are taken to be real, no objective selections of individual branches take place. Furthermore, as we have seen above, proponents of the DWE approach explicitly emphasise that no probabilities of measurement outcomes can be extracted from branch counting. Therefore, every pattern of outcomes of a quantum experiment that is not strictly forbidden by the laws of quantum mechanics is fully consistent with the DWE scheme and thus fully plausible at an objective level.

Proponents of the DWE scheme assert, however, that their approach to quantum mechanics implies the use of the Born rule in bets on empirical outcomes since no other betting behaviour is rational. This subjective implementation of quantum probability in some sense amounts to the claim that the Everettian approach makes roughly the same predictions about the outcome of quantum experiments as QM. In Chapter 6 of his book Wallace (2012), David Wallace explicitly asserts that the 'predictions' of DWE provide the basis for falsifying Everettian QM in the face of evidence that contradicts them. In his terms, Wallace claims that DWE has an answer to the 'unknown theory problem'. In the following, we shall argue that this assertion is mistaken.

Let us imagine that recent quantum experiments had provided empirical data that were significantly incompatible with the statistical predictions of QM. By this we mean experimental results which did not violate the nonprobabilistic laws of quantum mechanics but which were assigned a vanishingly small probability by the theory. A consistent (and diverse) sequence of such experimental results would eventually have lead the scientific community to accept that QM had been refuted.

How would the DWE scheme have been affected by the described empirical evidence? In principle, one could hope to implement falsification at two levels.

First, one might think that the collected data falsified the claim that one should use the Born rule in Everettian quantum mechanics. That is, the data might not suggest the falsity of Everettian QM per se but rather 
the inadequacy of the decision theoretic argument that implies the use of the Born rule. This does not work, however, since, according to DWE, the decision theoretic analysis itself does not rely on empirical data at all. It is based on the assumption of the truth of Everettian QM and introduces a set of allegedly cogent and indubitable general principles of rationality. Logical analysis then leads to the statement that applying the Born rule is the only rational betting behaviour for an agent in a branch of the Everettian world. The logical analysis seems unassailable and clearly cannot be refuted by empirical evidence. The assumed rationality principles are more contentious and have indeed been criticized by a number of observers Price (2010); Kent (2010) as arbitrary and unjustified. The exponent of DWE, however, in order not to abandon those principles and thereby the entire subjectivist approach from the start, must uphold them even in the face of false predictions made on their basis in the context of some scientific theory. If she was ready to take them to be empirically refuted as soon as the first scientific anomaly has been derived on their basis, they would be downgraded to empirical speculations and therefore become utterly useless.

This means, that one can only hope to find falsification of DWE with respect to structural elements of Everettian QM itself. But this leads us back to an analysis of the objective structure of Everettian QM. We know already that the DWE scheme does not provide objective probabilities for outcomes of experiments and therefore cannot be refuted by experimental data on that basis (provided that data is not strictly forbidden by the laws of quantum mechanics). We thus have no basis for the claim that structural elements of Everettian QM are falsified by the given data either. We must conclude that the considered data do not falsify Everettian QM at all.

At first sight, it may seem paradox that DWE 'predicts' data but empirical results which contradict those predictions do not falsify the theory. The situation becomes clear once one compares conventional predictions with 'predictions' as deployed in DWE based on a reconstruction in terms of hypothetico-deductivism. In conventional cases, the (statistical distribution of) empirical data to be found in experiments can be deduced from the theory. A contradiction between predicted and observed data thus implies that either the statement specifying the collected data or the theory is false. DWE, to the contrary, only asserts that statements about the rationality of betting behaviour can be deduced from Everettian QM. No deductive step is available that leads from the statement that only betting on a specific statistical data distribution is rational to the statement that this distribution will in 
fact be found in experiments. Therefore, the observation of contradicting data does not imply that either the theory or the statement about observed data is false. No falsification can occur.

In order to characterise the kind of fallacy involved in DWE reasoning, let us look at a toy example that has nothing to do with quantum physics. Let us assume a slightly modified form of Pascal's wager. Imagine that man has studied religion quite a lot and came up with the following heaven-theory: if there is life after death, the one who bets on it before death will get a million Pounds start capital in afterlife (heaven seems to be British) while the one who bets against it will get just one Pound. (Agnostics get nothing.) However, the chances that afterlife exists are just one over a million. Since no money can be received by the agent if afterlife does not exist, the only rational betting behaviour in the given scenario is betting on the existence of afterlife. Still, once the voice of god at the time of death tells you: 'sorry, no afterlife', your last thought should not be: 'So heaven-theory was false.' It should rather be: 'Given heaven-theory, I'm not surprised. After all, there is no logical implication that leads from the specification of rational betting behaviour to factual statements on the subject of betting.'

DWE thus gives us no justification at all for withdrawing the predictions according to the Born rule in the face of data that disagrees with the Born rule. The objective implications of DWE do not involve the Born rule and thus cannot be at variance with the new data; and the predictions extracted at the subjective level which do involve the Born rule are not based on empirical data and therefore cannot be refuted by it. We thus must conclude that DWE would not have been refuted by a large class of data that would have refuted QM. The form of prediction provided by DWE does not amount to scientific prediction as defined by refutability by data that is at variance with the prediction. DWE thus does not meet the standard of scientific viability set by $\mathrm{QM}^{4}$

The defender of DWE might try to rescue the general DWE scheme by making a minor amendment. They might introduce an additional rationality principle $\mathrm{P}$ that consists in the requirement that bets on future outcomes may only be placed as long as they do not stand in conflict with predictions based

\footnotetext{
${ }^{4}$ Note, in particular, that the presented conclusion blocks Occham's razor arguments for favouring Everettian QM over other interpretations of QM. If the Everett interpretation does not meet the standards of empirical viability of canonical QM, Occam's razor is no argument for favouring it over the latter.
} 
on cogent inductive reasoning. Once principle $\mathrm{P}$ is added, the defender of DWE might argue, bets according to the Born rule are not rational any more in the presence of significant empirical deviations from QM. The viability of the DWE predictions thus can be refuted by empirical data after all.

But rather than solving the problem, this step shows the precarious character of the decision theoretic argument even more clearly. Let us assume that recent quantum experiments showed a specific pattern of deviations from QM predictions. To have a nice simple example, let us imagine that one found a certain radioactive isotope that decayed according to a specifiable statistical rule $\mathrm{R}$ that was at variance with the Born rule. The extended rationality conditions would imply that there is no rational betting behaviour with respect to the decay of that isotope. The only rational option based on the DWE rationality conditions would be betting according to the Born rule; but principle $\mathrm{P}$ renders this strategy rationally excluded in the given case.

Any agent who followed basic principles of scientific reasoning and had the slightest experience in betting, however, would judge the observed pattern of incoming data based on the available background information and then know how to bet accordingly. Agents believing in Everettian QM in particular would adhere to the following line of reasoning: Everettian QM does not make objective predictions which contradict the observed pattern of data; on these grounds there is no reason for being less convinced of Everettian QM after the data than before; the data allows for immediate inductive predictions concerning the outcomes of future experiments based on the rule R; therefore, one should bet accordingly. Abstaining from this strategy would contradict the principle of inductive reasoning and therefore would be at variance with scientific thinking. In other words, the DWE rationality principles are incompatible with scientific thinking and therefore cannot be accepted as a foundation of scientific reasoning. Betting must always be carried out according to inductive reasoning. As long as that strategy is consistent with the DWE principles of rationality, the latter can be upheld. As soon as it differs from them, the DWE principles can only be defended at the price of abandoning scientifically implied forms of reasoning.

To conclude, the subjectivist approach of DWE does not make Everettian quantum mechanics a viable description of our world. Its failure in that respect can be stated in two different ways. First, if one endorses the rationality principles stated by DWE as universally valid, the DWE approach to Everettian QM does not meet the standards of empirical viability set by QM. And second, if one understands the principle of inductive reasoning as a core 
element of rationality, the rationality principles suggested by DWE become irrelevant because they can only be upheld as long as the betting strategy they imply is being enforced anyway by the principle of inductive inference.

\section{Branches, Weights, and Evidence}

\subsection{The Greaves-Myrvold approach}

The final argument of Section $\S 3$ has demonstrated that DWE does not provide a satisfactory mechanism of confronting Everettian QM with empirical evidence. In this section we will consider an approach towards subjectivist Everettian QM that is specifically designed to address the problem of empirical evidence. This approach, due to Greaves and Myrvold (2010) shares with the DWE scheme the understanding that the statistical character of quantum mechanics cannot be established at an objective level but at the same time accepts the need to infer the Born rule from empirical data. ${ }^{5}$ To that end, Greaves and Myrvold, use a decision theoretic argument very much in the style of Deutsch and Wallace, but within a different context: that of theory confirmation by empirical evidence.

Greaves and Myrvold emphasise that quantum physicists must at any rate account for the observed statistical pattern of quantum measurements and, as scientists, must inductively apply that information in order to predict future outcomes. (Thereby, they acknowledge the additional 'rationality principle' formulated at the end of Section §3.) On that basis, quantum physicists observe that the relative frequency data they collect are in agreement with the Born rule and therefore accept that rule. Greaves and Myrvold argue that the adoption of the Born rule should be motivated by the Everettian on the described evidential basis and be implemented within the framework of an Everettian branching structure by attributing weights between 0 and 1 to individual branches accordingly. These weights correspond to the predicted probabilities of measurement outcomes but do not constitute probabilities themselves. Rather, they just serve as primitive indicators of quantitative predictions. Thus, the Greaves-Myrvold strategy involves the substitution

\footnotetext{
${ }^{5}$ The reader is referred to Greaves (2007) for an interesting precursor to the Greaves and Myrvold analysis. The difference between that approach and the later treatment considered here are not significant for our purposes.
} 
for - rather than explanation of - the usual connection between observed evidence and probabilistic dynamical structure. The claim is that:

[An] agent will regard relative frequency data from repeated experiments as informative about values of branch weights in exactly the same way that, on the usual view, they are informative about chances of outcomes. (p.7)

and that:

[G]iven reasonable constraints on an epistemic agent's preference between wagers, she will act as if she believes that there are physical branch weights, analogous to physical chances, that can be estimated empirically in the same way that chances are, and that observation of events to which a theory assigns high branch weight boosts rational credence in a branching theory in the same way that observation of events to which a theory assigns high chance boosts rational credence in a chance theory. (p.28)

The approach is subjectivist because it does not provide an interpretation of the branch weights within the context of the objective Everettian branching structure. The branch weights only acquire meaning with respect to an individual agent in an individual branch, where they denote the agent's probabilistic expectations qua betting behaviour. Significantly, the probabilistic expectations of the agent are not understood as being in any way connected to the occurrence or not of the relevant outcomes since, as in any Everettian style picture, all outcomes will occur. This means that, as noted by Albert (2010), under the Greaves-Myrvold analysis, "the business of deciding how to bet is just a matter of maximising the payoffs on those branches that I happen to care most about' and has 'nothing whatsoever to do with guessing at whether or not [an event] is going to occur' (p.364) [italics in the original]. This facet of the approach is certainly unusual - customarily we would expect any account of scientific theory confirmation to be based upon the theories ability to make reliable predictions of the occurrence of events rather than robust guidelines to branch weight based betting. However, it would be unfair to see this crucial shift as an underhand move: as seen from the quotes above Greaves and Myrolvd are at pains to be explicit about their radical departure from our usual notion of confirmation - and it surely is not legitimate to simply reject the scheme simply on the grounds 
of its strangeness. Nevertheless, questions as to whether or not Greaves and Myrvold have stretched the concepts of betting and evidence too far past the customary meanings to remain viable, certainly constitute a valid line of objection - and in this sense there is certainly a case to answer in the face of the (rather trenchant) criticisms of Albert (2010). For discussion of such issues as well as possible adaptations of Greaves-Myrvold scheme in the face of them, see Tappenden (2011). However, for the purpose of this paper and in continuity with our discussion of DWE above - we will concede the internal conceptual coherence of the scheme in order to focus upon external issues; in particular the legitimacy of the Greaves-Myrvold approach within the Everettian family.

\subsection{Empirically viable but no longer viably Everettian}

Greaves and Myrvold take their approach to be consistent with DWE, and envisage their work as a supplement to that of Deutsch and Wallace which provides a framework for theory confirmation in a branching (although not necessarily Everettian) universe. This understanding is in agreement with Wallace's own perspective, who points out at the end of Chapter 4 in Wallace (2012) that the decision theoretic argument may be seen as a more thorough explanation of the validity of the Born rule, which could in principle also be inferred from the collected data based on inductive reasoning. Essentially, what was demonstrated in Section $\S 3$ of this paper is that the rationality criteria suggested by DWE cannot be reconciled with a full commitment to inductive reasoning. Our argument thus contradicts Wallace's claim that 'specific predictions within Everettian quantum mechanics can be confirmed or refuted in the same way as for non-Everettian theories' (Wallace, 2012, p.210). Inversely, on our account, an agent deliberating within the framework of Greaves and Myrvold can not simultaneously operate within the framework of DWE since their fundamental decision principles will conflict. Let us therefore proceed to investigate how the Greeves-Myrvold approach fares as an alternative approach to Everettian QM in its own right.

Significantly for our purposes, the account of Greaves and Myrvold would appear to have a substantial empirical advantage over the DWE approach. According to Greaves and Myrvold the overall scheme that should be held by the quantum physicist is constituted by the objectively stated structure of Everettian QM in conjunction with the empirically established attribution of branch weights according to the Born rule. That overall scheme is empirically indistinguishable from QM with respect to the currently available data. 
Moreover, data that deviated significantly from the Born rule would have refuted the Greaves-Myrvold scheme in the same way as it would have refuted QM. In this sense, Greaves and Myrvold, unlike Deutsch and Wallace, have managed to formulate a theory that meets the standard of empirical viability set by QM. They have done so, however, at a very high cost. Let us take one step back to put the move by Greaves and Myrvold into context.

Everettian QM was originally proposed in order to keep the equations of quantum mechanics free from additional principles which established the relation between the wave function and the stochastic character of empirical data - and its modern defenders take this feature to be one of its principal strengths. Canonical 'Copenhagen' QM introduces the collapse of the wave function and then relates the statistics of empirical data to the stochastic characteristics of the collapse. Bohmian QM introduces (non-local) hidden parameters and relates the statistics of empirical data to the initial conditions of particles. Through the Everettian approach, it is claimed that quantum mechanics can do without any additions. The statistics of empirical data should follow just from the pure unitary dynamics of the wave function. This central Everettian claim thus is a very significant one and, if vindicated, would constitute a clear strengthening of the status of quantum mechanics.

As we have seen, however, a straightforward deduction of the Born rule from the dynamics of the wave function has turned out not to be achievable in the context of a many worlds type approach. It is for this reason Deutsch and Wallace made the step from the objective to the subjective level. They said: even if we cannot deduce the Born rule from what Everettians take to be the objective many worlds structure of the universe, we can show that applying the Born rule is not a contingent choice for an agent in one of the branches of the universe. It is the only rational strategy available and therefore does not have to be derived at an objective structural level. Deutsch and Wallace therefore retain the strength of Everettian QM as an approach that does not introduce any physical posits beyond the equations of quantum mechanics. We have seen in the previous section, however, that this approach fails because, in the end, it does not lead to an empirically viable theory.

Greaves and Myrvold suggest that we just take into account the empirical data and establish the Born rule on that basis subjectively. In doing so, however, they surrender a core claim of Everettian QM: that we can deduce the full phenomenology from the dynamics of the wave function without any additional assumptions. For their scheme to work, Greaves and Myrvold must 
make the additional assumption that our observations in quantum physics are in accordance with Born weights attributed to branches. The introduction of this assumption unquestionably amounts to supplementing the bare formalism with additional structure and thus a failure to retain the prima facie most attractive feature of Everettian quantum mechanics. Under Greaves and Myrvold's approach, the equations of quantum mechanics can no longer be claimed to yield their own interpretation.

Furthermore, explanatorily the additional structure they introduce is remarkably uninteresting. Whereas, for example, in dynamical collapse or Bohmian models, additional structure is introduced such that a plausible basis for the statistical characteristics of our observations is rooted in the structural characteristics of quantum mechanics, Greaves and Myrvold merely introduce a module that is entirely phenomenal in character and stays independent of all objective structural aspects of the theory. The phenomenal implications of this module could in fact be tuned at will in order to fit the empirical data without affecting our understanding of the theory's objective structure. If an agent observed a different statistics than the one implied by the Born rule, then they could fix the weights accordingly and thus establish that statistics as a rule; if they observed no discernible statistical pattern at all, then they could conclude that the weights do not follow any discernable pattern and thus refrain from predicting anything - all without changing the meaning of any objective structural properties of Everettian QM. Greaves and Myrvold in effect say: we just don't know why the Born rule applies, so let us simply take it as a primitive fact that it does. Given that most of what is empirically interesting about quantum mechanics is related to the Born rule, this amounts to a capitulation. In order to make the subjectivist approach sensitive to empirical data, Greaves and Myrvold deem it necessary to turn it into an empty shell.

This point can be illustrated by means of a simple example from classical statistical physics. Consider an ideal gas at a moderate temperature and close to thermal equilibrium; and consider an idealised experiment where the velocities of individual gas molecules are measured. Given a certain limited degree of accuracy, such measurements would lead to the empirical observation that the molecules obey a certain probability distribution - i.e. the Maxwell-Botzmann distribution for the given temperature. The standard classical mechanical description of such a system according to kinetic theory would provide an account of the observed distribution in terms of structural features of the theory itself - i.e. the particular statistical distri- 
bution observed is rooted in the theoretical framework used. Observation of a statistical distribution at odds with that of Maxwell and Boltzmann would constitute a refutation of kinetic gas theory.

Let us then consider a scheme SKT which consists of certain of the principal assumptions of kinetic theory but does not have sufficient theoretical structure to derive the full statistical predictions of the theory (Newton's force law is missing for example!). Now, let us allow for SKT to contain a Maxwell-Botzmann 'phenomenological module' - analogous to the Born 'phenomenological module' that Greaves and Myrvold attached to Everettian theory - such that we take into account the relevant observed empirical data in order to establish the Maxwell-Botzmann distribution subjectively as a belief enforced by rationality constraints upon an inductive scientist. In such circumstances one would be able to successful predict the behaviour of the system based upon SKT just as if it were a substantive physical theory. Moreover, by our criteria $\mathbf{I}_{2}$, SKT would meet the standard of empirical viability set by kinetic theory since any data that could have been collected in experiments which have been carried out in the past and would have refuted kinetic theory would also have refuted SKT. ${ }^{6}$ However, clearly SKT is of almost no scientific value precisely because - as in the Greaves-Myrvold case - it relies upon a phenomenological module that is entirely independent of all objective structural aspects of kinetic theory and could in fact be tuned at will in order to fit the empirical data without affecting our understanding of the theory's objective structure.

Of course, the Geaves-Myrvold approach to quantum theory differs from the SKT approach to kinetic theory by constituting a response to a specific interpretational problem. However, the essential features of both schemes are the same: the statistical predictions are established based upon an inductive 'module' rather than integrated theoretical structure. Although such strategies may be both coherent and by our lights empirically viable, as contributions to our understanding of the world they are almost entirely without merit; and improving our understanding of the world arguably is what interpretations of quantum mechanics should be aiming at.

\footnotetext{
${ }^{6}$ Refutation of a theory in the given statistical context means that the probability that the observed data pattern is generated by the theory in question lies below a specified very small value. In high energy physics, to give an example, a theory counts as refuted if it disagrees with the data at $5 \sigma$ confidence level.
} 


\section{Conclusion}

The Deutsch-Wallace-Everett approach fails to provide an empirically viable form of quantum mechanics. Everettian QM based on a subjective implementation of the Born rule as suggested by Deutsch and Wallace would be empirically refutable to a far lesser degree than QM per se. Moreover, the rationality conditions introduced by Deutsch and Wallace would be at variance with scientific reasoning when confronted with data that violates QM by being at variance with the Born rule.

Unlike the DWE-scheme, the approach of Greaves and Myrvold, which relies on extracting the Born rule at a subjective level from empirical data rather than from rationality principles, is capable of providing an empirically viable form of quantum mechanics. This approach, however, jettisons much of what is interesting about interpretations of QM in general (it does not offer any deeper level explanation of the Born rule at all) and about Everettian QM in particular (it gives up the Everettian aim to avoid additional physical posits beyond the equations of quantum mechanics). Thus, the Greaves-Myrvold approach does not look like a promising step towards a deeper understanding of quantum physics.

The described dilemma between loosing predictive power in the decision theoretical framework on the one hand, and deploying inductive reasoning in a primitive form without deeper entrenchment within the conceptual principles of quantum mechanics on the other, seems to be unavoidable once a subjectivist approach towards Everettian quantum physics has been chosen. Anchoring part of the predictive power of the theory at the subjective level isolates that part from the theory's objective structure and therefore blocks all chances of relating the corresponding results of inductive inference to that structure. Decision theoretic arguments, however, are not data-based and therefore cannot replace inductive reasoning as a basis for empirically testable scientific predictions. In our understanding, the described dilemma suggests that, to the extent the Everettian approach may be seen as promis-

ing, its future fertility is likely to be found at the level of objective Everettian structure. 


\section{Acknowledgements}

We would like to thank Stephan Hartmann and Jeffrey Barrett for valuable discussion, and a number of referees for providing feedback which aided in the development of the paper. Work on this paper also greatly benefited from the productive environment provided initially by the Tilburg Center for Logic, General Ethics, and Philosophy of Science, and more recently by the Munich Center for Mathematical Philosophy. The paper was partially funded (R.D.) by the Austrian Research Fund (FWF) project: P22811-G17. We both acknowledge the support of the Alexander von Humboldt foundation.

\section{References}

Albert, D., 2010. Probablity in the everett picture. In: Saunders, S., Barrett, J., Wallace, D., Kent, A. (Eds.), Many Worlds? Everett, Quantum Theory, and Reality. Oxford Univeristy Press, Ch. 11, pp. 354-368.

Baker, D. J., 2007. Measurement outcomes and probability in everettian quantum mechanics. Studies In History and Philosophy of Science Part B: Studies In History and Philosophy of Modern Physics 38 (1), 153 - 169. URL http://www.sciencedirect.com/science/article/pii/S1355219806000694

Barrett, J. A., 2011. On the faithful interpretation of pure wave mechanics. The British Journal for the Philosophy of Science 62 (4), 99-123.

Deutsch, D., 1999. Quantum theory of probability and decisions. Proceedings of the Royal Society of London. Series A: Mathematical, Physical and Engineering Sciences 455 (1988), 3129-3137.

URL http://rspa.royalsocietypublishing.org/content/455/1988/3129. abstract

Greaves, H., 2007. On the everettian epistemic problem. Studies In History and Philosophy of Science Part B: Studies In History and Philosophy of Modern Physics 38 (1), 120-152.

Greaves, H., Myrvold, W., 2010. Everett and evidence. In: Saunders, S., Barrett, J., Kent, A., Wallace, D. (Eds.), Many Worlds? Everett, Quantum Theory, and Reality. Oxford Univeristy Press, Ch. 9, pp. 264-304. 
Hemmo, M., Pitowsky, I., 2007. Quantum probability and many worlds. Studies In History and Philosophy of Science Part B: Studies In History and Philosophy of Modern Physics 38, 333-350.

Kent, A., 2010. One world versus many: The inadequacy of Everettian accounts of evolution, probability, and scientific confirmation. In: Saunders, S., Barrett, J., Wallace, D., Kent, A. (Eds.), Many Worlds? Everett, Quantum Theory, and Reality. Oxford Univeristy Press, Ch. 10, pp. 307-355.

Lewis, P., 2006. Uncertainty and probability for branching selves. Studies In History and Philosophy of Science Part B: Studies In History and Philosophy of Modern Physics 38 (1), 1/14.

Price, H., 2010. Decisions, Decisions, Decisions: Can Savage Salvage Everettian Probability? In: Saunders, S., Barrett, J., Kent, A., Wallace, D. (Eds.), Many Worlds? Everett, Quantum Theory, and Reality. Oxford Univeristy Press, pp. 369-391.

Rae, A. I., 2009. Everett and the Born rule. Studies In History and Philosophy of Science Part B: Studies In History and Philosophy of Modern Physics 40 (3), $243-250$.

URL http://www.sciencedirect.com/science/article/pii/S1355219809000306

Tappenden, P., 2011. Evidence and uncertainty in everett's multiverse. The British Journal for the Philosophy of Science 62 (4), 693-709.

Wallace, D., 2007. Quantum Probability from Subjective Likelihood: improving on Deutsch's proof of the probability rule. Studies In History and Philosophy of Science Part B: Studies In History and Philosophy of Modern Physics 38 (2), 311-332.

Wallace, D., 2009. A formal proof of the born rule from decision-theoretic assumptions. ArXiv e-prints http://arxiv.org/abs/0906.2718v1.

URL http://arxiv.org/abs/0906.2718v1

Wallace, D., 2012. The Emergent Multiverse. Oxford Univeristy Press.

Zurek, W. H., May 2005. Probabilities from entanglement, Born's rule $p_{k}=\mid$ $\left.\psi_{k}\right|^{2}$ from envariance. Phys. Rev. A 71, 052105.

URL http://link.aps.org/doi/10.1103/PhysRevA.71.052105 
Zurek, W. H., 2010. Quantum jumps, Born's rule, and objective reality. In: Saunders, S., Barrett, J., Kent, A., Wallace, D. (Eds.), Many Worlds? Everett, Quantum Theory, and Reality. Oxford Univeristy Press, Ch. 13, pp. 409-432. 